\title{
Spectroscopic indications of polaronic behavior of the strong spin-orbit insulator $\mathrm{Sr}_{3} \mathrm{Ir}_{2} \mathrm{O}_{7}$
}

\author{
P. D. C. King, ${ }^{1,2,3,{ }^{*}}$ T. Takayama, ${ }^{4}$ A. Tamai,${ }^{1,5}$ E. Rozbicki, ${ }^{1}$ S. McKeown Walker, ${ }^{1,5}$ M. Shi, ${ }^{6}$ L. Patthey, ${ }^{6,7}$ R. G. Moore, ${ }^{8}$ \\ D. Lu, ${ }^{9}$ K. M. Shen, ${ }^{2,3}$ H. Takagi, ${ }^{4,10}$ and F. Baumberger ${ }^{1,5,6, \dagger}$ \\ ${ }^{1}$ SUPA, School of Physics and Astronomy, University of St. Andrews, St. Andrews, Fife KY16 9SS, United Kingdom \\ ${ }^{2}$ Kavli Institute at Cornell for Nanoscale Science, Ithaca, New York 14853, USA \\ ${ }^{3}$ Laboratory of Atomic and Solid State Physics, Department of Physics, Cornell University, Ithaca, New York 14853, USA \\ ${ }^{4}$ Department of Physics, University of Tokyo, Hongo, Tokyo 113-0033 \\ ${ }^{5}$ Département de Physique de la Matière Condensée, Université de Genève, 24 Quai Ernest-Ansermet, 1211 Genève 4, Switzerland \\ ${ }^{6}$ Swiss Light Source, Paul Scherrer Institut, CH-5232 Villigen PSI, Switzerland \\ ${ }^{7}$ SwissFEL, Paul Scherrer Institut, CH-5232 Villigen PSI, Switzerland \\ ${ }^{8}$ Stanford Institute for Materials and Energy Sciences, SLAC National Accelerator Laboratory, 2575 Sand Hill Road, \\ Menlo Park, California 94025, USA \\ ${ }^{9}$ Stanford Synchrotron Radiation Lightsource, SLAC National Accelerator Laboratory, 2575 Sand Hill Road, \\ Menlo Park, California 94025, USA \\ ${ }^{10}$ Magnetic Materials Laboratory, RIKEN Advanced Science Institute, Wako, Saitama 351-0198, Japan \\ (Received 31 January 2013; revised manuscript received 11 May 2013; published 24 June 2013)
}

\begin{abstract}
We investigate the bilayer Ruddlesden-Popper iridate $\mathrm{Sr}_{3} \mathrm{Ir}_{2} \mathrm{O}_{7}$ by temperature-dependent angle-resolved photoemission. At low temperatures, we find a fully gapped correlated insulator, characterized by a small charge gap and narrow bandwidths. The low-energy spectral features show a pronounced temperature-dependent broadening and non-quasiparticle-like Gaussian line shapes. Together, these spectral features provide experimental evidence for a polaronic ground state. We observe similar behavior for the single-layer cousin $\mathrm{Sr}_{2} \mathrm{IrO}_{4}$, indicating that strong electron-boson coupling dominates the low-energy excitations of this exotic family of $5 d$ compounds.
\end{abstract}

DOI: 10.1103/PhysRevB.87.241106

PACS number(s): 71.27.+a, 71.30.+h, 71.38.-k, 79.60.Bm

The strong spin-orbit interaction in the $5 d$ shell is predicted to stabilize a variety of exotic ground states in iridium-based transition-metal oxides, including Mott insulators, ${ }^{1-3}$ Weyl semimetals, ${ }^{4}$ correlated topological insulators, ${ }^{5-8}$ and spintriplet superconductors. ${ }^{9}$ Moreover, iridates were recently proposed as an analog of the cuprates, and as such, a potential platform for engineering high-temperature superconductivity. ${ }^{10}$ This initially appears surprising given the weak influence of electron correlations expected for spatially extended $5 d$ orbitals. Nonetheless, $\mathrm{Sr}_{2} \mathrm{IrO}_{4}$ and $\mathrm{Sr}_{3} \mathrm{Ir}_{2} \mathrm{O}_{7}$, which both host partially filled $5 d$ shells, are found to be insulating. ${ }^{11,12}$ For $\mathrm{Sr}_{2} \mathrm{IrO}_{4}$, this was recently attributed ${ }^{1,2}$ to a reconstruction of the underlying electronic structure by a cooperative interplay of structural distortions and, crucially, the strong spin-orbit coupling, leaving a half-filled $J_{\text {eff }}=1 / 2$ band that is sufficiently narrow that even moderate correlation strengths can drive a Mott transition. This $J_{\text {eff }}=1 / 2$ space can be mapped onto a pseudospin-1/2 Hubbard model, providing the analogy to the cuprates. ${ }^{10}$ Microscopically, however, the similarity of the insulating ground states in these parent compounds remains an open question. The orbital configuration is $5 d^{5}$ in $\mathrm{Sr}_{n+1} \mathrm{Ir}_{n} \mathrm{O}_{3 n+1}$ as compared to $3 d^{9}$ in the cuprates. Spin-orbit interactions play an important role in the former, ${ }^{1,2,13-20}$ while the behavior of the latter is dominated by strong electron correlations. Even the range of validity of the strong spin-orbit $J_{\text {eff }}=1 / 2$ Mott picture for the iridates, on which links to the cuprates have been based, remains an open question. ${ }^{21-25}$ As such, detailed studies of the low-energy electronic excitations of iridates are required to elucidate the nature of the complex many-body ground states of these compounds.

Indeed, kinetic, Coulomb, crystal-field, and spin-orbit energy scales are all of similar magnitude in the iridates, potentially leading to the close proximity of sev- eral competing ground states. For example, optical conductivity measurements revealed a metal-insulator transition (MIT) upon increasing dimensionality through the layered Ruddlesden-Popper series $\operatorname{Sr}_{n+1} \mathrm{Ir}_{n} \mathrm{O}_{3 n+1},{ }^{3}$ with the conducting three-dimensional end member predicted to be an exotic semimetal. $^{26}$ The $n=2$ compound, which crystallizes in an orthorhombic structure [space group Bbca (Ref. 12)], is composed of Ir-O bilayers separated by $\mathrm{Sr}-\mathrm{O}$ blocking layers. As for the single-layer compound $\mathrm{Sr}_{2} \mathrm{IrO}_{4}$, the $\mathrm{IrO}_{6}$ octahedra are slightly elongated along the $c$ axis, and exhibit a large (staggered) in-plane rotation $\left(\sim 11^{\circ}\right.$ in $\left.\operatorname{Sr}_{3} \operatorname{Ir}_{2} \mathrm{O}_{7}\right)$. The bilayer structure is expected to result in an intermediate dimensionality and physical properties close to the border line between the insulating single-layer compound $\mathrm{Sr}_{2} \mathrm{IrO}_{4}$ and the (semi)metallic three-dimensional end member $\mathrm{SrIrO}_{3}{ }^{3}$ promising new insights into the nature of the MIT and the unconventional insulating ground states of iridates.

Here, we study its low-energy electronic structure by angleresolved photoemission (ARPES). We find a small charge gap to a weakly dispersive band, reminiscent of a $J_{\text {eff }}=1 / 2$ lower Hubbard band, although with other nearby dispersive bands that complicate this picture. The lowest-energy excitations show a non-quasiparticle-like Gaussian line shape and a strong temperature dependence of the line width. These spectral properties are notably similar to manganites ${ }^{28}$ and lightly doped cuprates ${ }^{27}$ and provide experimental evidence for a polaronic ground state in layered $5 d$ iridates.

ARPES measurements were performed at the Surface and Interface Spectroscopy (SIS) beamline of the Swiss Light Source and beamline V-4 of the Stanford Synchrotron Radiation Lightsource, and using a laboratory He-lamp system. Measurements were made using photon energies between 16 and $120 \mathrm{eV}$ and both Scienta R4000 and SPECS Phoibos 

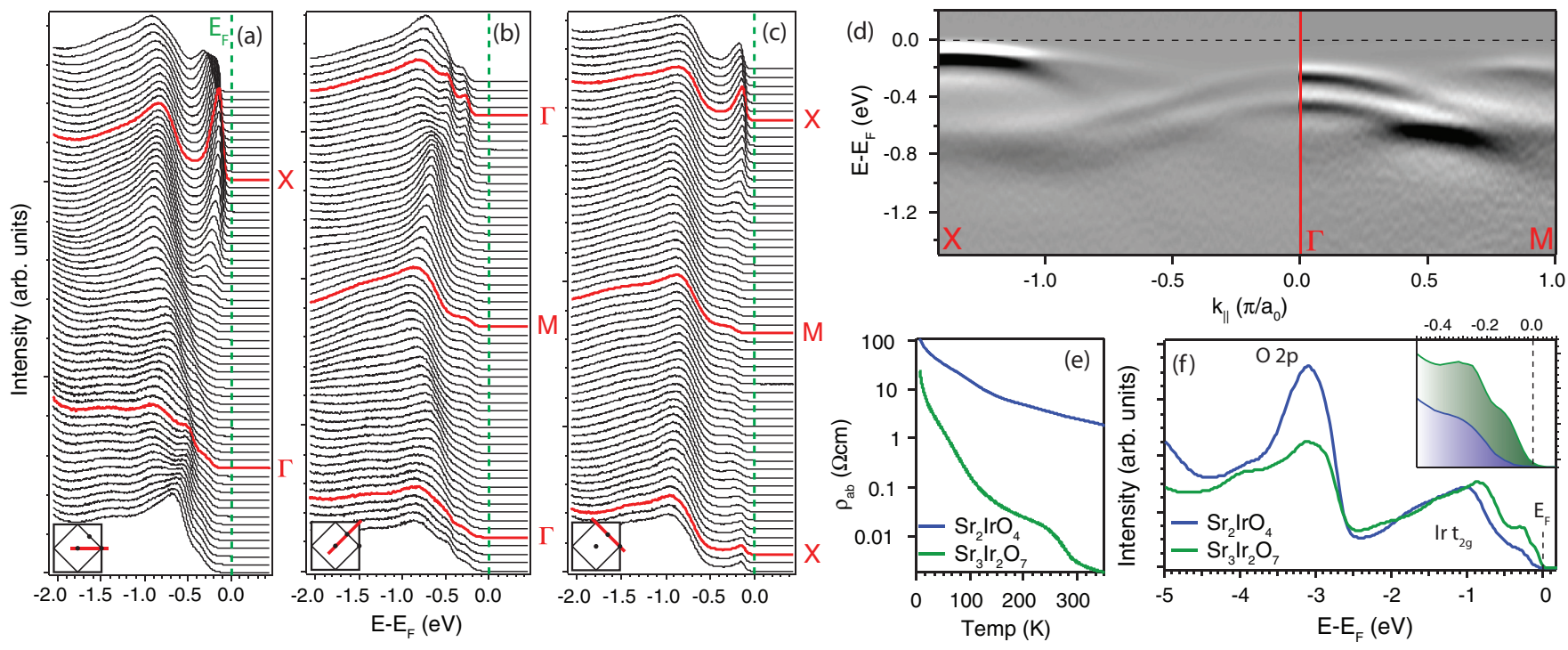

FIG. 1. (Color online) Low-energy electronic structure of $\operatorname{Sr}_{3} \mathrm{Ir}_{2} \mathrm{O}_{7}$, measured with a photon energy of $40 \mathrm{eV}$ at $50 \mathrm{~K}$ along the (a) $\Gamma$ - $X$, (b) $\Gamma$ $M$, and (c) $M-X$ high-symmetry directions of the Brillouin zone (see insets). (d) Second-derivative image plots clearly show rapid dispersion of multiple bands along $X-\Gamma-M$. Temperature-dependent resistivity and valence band photoemission are compared for the bilayer (green) and single-layer (blue) compounds in (e) and (f), respectively. A magnified view of the near- $E_{F}$ valence band emission is shown inset in (f).

225 hemispherical analyzers. The total experimental energy resolution was below $20 \mathrm{meV}$, and the sample temperature was varied between 25 and $350 \mathrm{~K}$. Single-crystal samples of $\mathrm{Sr}_{3} \mathrm{Ir}_{2} \mathrm{O}_{7}$ and $\mathrm{Sr}_{2} \mathrm{IrO}_{4}$ were flux-grown. These were cleaved in situ at pressures better than $5 \times 10^{-11}$ mbar.

The electronic structure of $\mathrm{Sr}_{3} \mathrm{Ir}_{2} \mathrm{O}_{7}$ measured at $50 \mathrm{~K}$ along high-symmetry directions, as well as its momentum dependence across the full Brillouin zone, is summarized in Figs. 1 and 2(a), respectively. ${ }^{29}$ We find that no bands intersect the Fermi level, with spectral weight tending to zero by the chemical potential. Detailed photon-energy-dependent measurements (not shown) reveal that this charge gap is robust throughout the full three-dimensional Brillouin zone. ${ }^{32}$ This identifies the low-temperature phase of $\mathrm{Sr}_{3} \mathrm{Ir}_{2} \mathrm{O}_{7}$ as a fully gapped insulator, consistent with the nearly exponential temperature dependence of its resistivity [Fig. 1(e)]. However, comparison of its angle-integrated spectra [Fig. 1(f)] to those of its single-layer counterpart reveals significantly enhanced spectral weight close to the Fermi level in the bilayer compound, as well as a much smaller charge gap.

Indeed, we observe dispersive features with well-defined peaks centered as little as $\sim 100 \mathrm{meV}$ below the Fermi level. The lowest-energy features are located at the $X$ point $[(\pi, 0)$ point of the tetragonal Brillouin zone], as in the single-layer compound. ${ }^{1}$ This is in qualitative disagreement with LDA + U calculations, even when spin-orbit interactions are included, ${ }^{3}$ which predict that the top of the valence band occurs at the zone center. Second-derivative plots [Fig. 1(d)] reveal that two bands disperse away from the band maximum at the $X$ point. We estimate that the upper band, which evolves into a weak shoulder near the $M$ point $[(\pi / 2, \pi / 2)$, Figs. 1(b) and 1(c)], disperses by only $\sim 150-200 \mathrm{meV}$ across the Brillouin zone, reflecting very narrow electronic bandwidths in this compound.

This appears broadly consistent with a narrow $J_{\text {eff }}=$ $1 / 2$ lower Hubbard band, as proposed for other iridates. ${ }^{1,33}$
In angle-integrated spectra of the single-layer compound [Fig. 1(f)], two dominant peaks at $\sim 0.25$ and $\sim 1 \mathrm{eV}$ are observed, which might be assigned as the origins of the $\alpha$ and $\beta$ transitions in optical conductivity. ${ }^{3}$ Such features, observed in several iridate oxides, have been attributed to

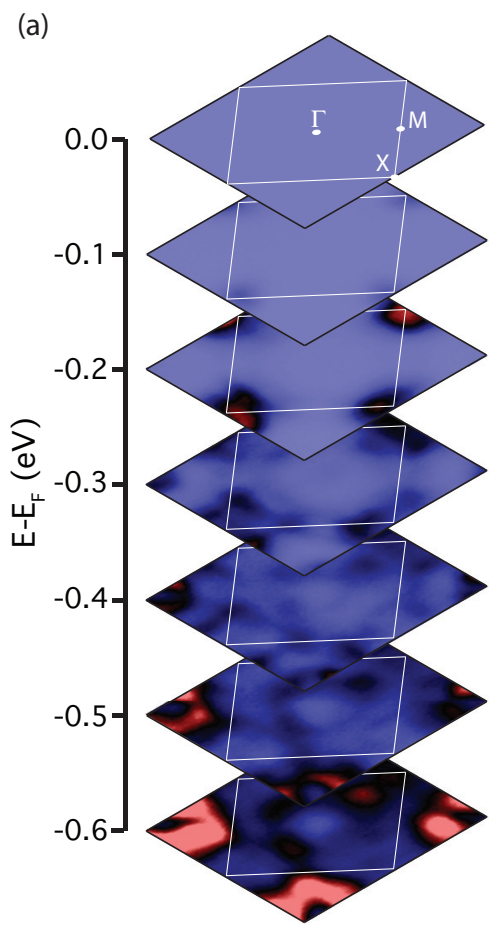

(b)
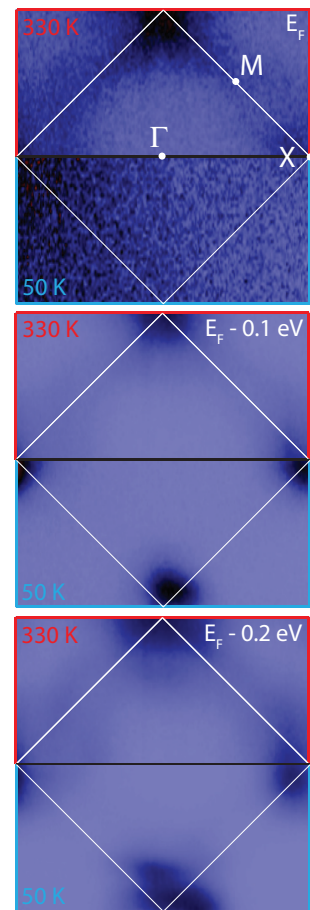

FIG. 2. (Color online) (a) Constant energy contours measured across the full Brillouin zone of $\mathrm{Sr}_{3} \mathrm{Ir}_{2} \mathrm{O}_{7}$ at $50 \mathrm{~K}(h v=70 \mathrm{eV})$ and normalized to the same total intensity for each energy. (b) Comparison of low-temperature $(50 \mathrm{~K})$ and high-temperature $(330 \mathrm{~K})$ maps at the Fermi level and 0.1 and $0.2 \mathrm{eV}$ binding energy, normalized to the highest intensity at that binding energy to maximize contrast. 
optical transitions to the upper Hubbard band from a single $J_{\text {eff }}=1 / 2$ lower Hubbard band and a separate $J_{\text {eff }}=3 / 2$ band, respectively. ${ }^{1,3,33,34}$ In contrast, the situation already appears more complex in angle-integrated spectra from $\mathrm{Sr}_{3} \mathrm{Ir}_{2} \mathrm{O}_{7}$, with additional peaks present at low binding energies compared to $\mathrm{Sr}_{2} \mathrm{IrO}_{4}$. This could reflect the presence of a non-negligible octahedral crystal-field splitting. ${ }^{22}$ Moreover, departures from an idealized $J_{\text {eff }}=1 / 2,3 / 2$ picture can be readily identified in angle-resolved spectra. For example, at the $X$ point, two well-separated peaks are observed in energy distribution curves (EDCs), which could naturally be assigned as distinct $J_{\text {eff }}=1 / 2$ and $3 / 2$ manifolds. However, significant dispersion is observed at other momenta, which presumably leads to small avoided crossings that are difficult to resolve experimentally, as well as substantial mixing of the $J_{\text {eff }}=1 / 2$ and $3 / 2$ states.

It is therefore an oversimplification to describe $\mathrm{Sr}_{3} \mathrm{Ir}_{2} \mathrm{O}_{7}$ as a prototypical $J_{\text {eff }}=1 / 2$ Mott insulator. This raises the question as to whether the insulating state results from some form of multiband Mott-like transition, or whether additional order parameters such as magnetism may play a crucial role, ${ }^{19,24,25}$ for example within a Slater-type picture. Consistent with previous measurements, ${ }^{19}$ we observe a pronounced drop in the resistivity of the bilayer compound at $\sim 280 \mathrm{~K}$ [Fig. 1(e)], concurrent with magnetic ordering. ${ }^{12}$ We find no sharp changes in the electronic structure measured by ARPES through this transition. Even at temperatures as high as $330 \mathrm{~K}$ [Fig. 2(b)], there is still only minimal spectral weight at the Fermi level. At energies below the Fermi level, the spectral weight across the Brillouin zone is dominated by the top of the hole-like bands at the $X$ point at both high [Fig. 2(b), top panels] and low [Fig. 2(b), bottom panels] temperatures. At the Fermi level itself, there appears to be a relatively higher contribution from states around the $M$ point in the higher temperature measurements. This could suggest a closing of the insulating charge gap from below $E_{F}$ at the $X$ point and above $E_{F}$ at the $M$ point, indicating a transition from an insulator to a semimetal with increasing temperature. This manifests as a very gradual increase in spectral weight at the Fermi level. A broad step in resistivity could still be explained in such a picture as a crossover from gapped transport at low temperatures to incoherent, electron-like carriers with low density above the closing of the Mott gap. However we note that, spectroscopically, this does not appear to occur as a sudden phase transition at $T_{N}$.

Instead, it appears to be driven by a substantial and continuous broadening of the spectral features with increasing temperature. Figure 3(a) shows the temperature dependence of EDCs at the $X$ point. At temperatures as low as $\sim 200$ $\mathrm{K}$, the pronounced peak visible at lower temperatures has broadened significantly into a weak hump-like feature. Indeed, the linewidth of EDCs at both the $X$ [Fig. 3(b)] and $\Gamma$ [Fig. 3(c)] points both increase with temperature at a rate as high as $0.3 \mathrm{meV} / \mathrm{K}$. This temperature evolution is almost identical for both warming and cooling of the same sample [Figs. 3(b) and 3(c)], ruling out sample aging as the origin of the observed broadening. Rather, it reflects a significant change in the lifetime of the initial state that is being probed in the ARPES, revealing a substantial temperature dependence of the many-body interactions. This is characteristic of strong

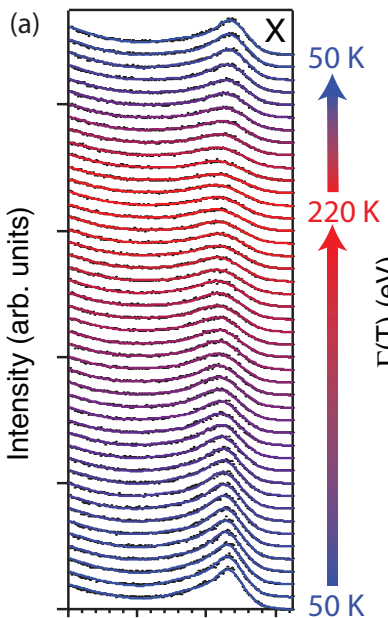

$\begin{array}{llll}-0.6 & -0.4 & -0.2 & 0.0\end{array}$

$$
\mathrm{E}-\mathrm{E}_{\mathrm{F}}(\mathrm{eV})
$$

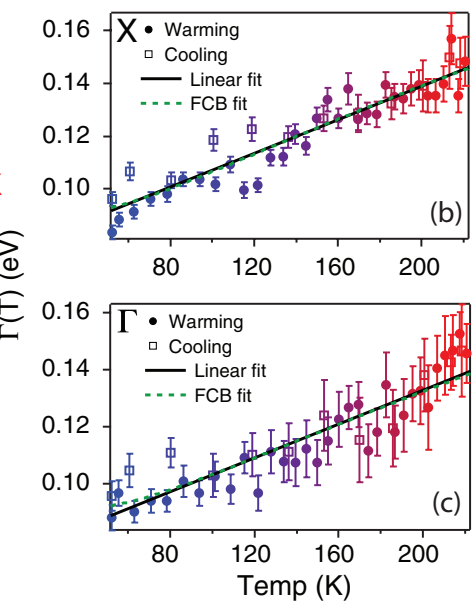

FIG. 3. (Color online) (a) Temperature dependence of EDCs (black dots) at the $X$ point. The spectra were all measured on a single sample, from bottom to top with increasing time, at a photon energy of $31 \mathrm{eV}$. Fits to two Gaussian peaks and a polynomial background are also shown (solid lines). The resulting extracted width $\Gamma(T)$ of the lowest-energy peak is shown in (b), and for equivalent measurements at the $\Gamma$-point in (c). These full data sets were fit by a linear slope (black lines) and by a model function to describe Franck-Condon broadening (green-dashed lines), as discussed in the main text.

electron-boson coupling, and usually indicative of a strong coupling to the lattice. Furthermore, the spectral features we observe are distinctly non-quasiparticle like. Instead of a narrow Lorentzian peak at the top of the valence band, ${ }^{35}$ we consistently observe broad features that can only be satisfactorily fit using Gaussian line shapes [Fig. 4(a)]. We stress that we experimentally ruled out insufficient energy resolution and sample charging as the origin of these spectroscopic signatures. The temperature dependence of the measured linewidths is also inconsistent with a defect-driven mechanism of their Gaussian line shapes. Moreover, similar (or even lower) defect densities would typically be expected for the structurally simpler and more robustly insulating single-layer $\mathrm{Sr}_{2} \mathrm{IrO}_{4}$ than for the bilayer compound, while we observe broader linewidths in the former as discussed below.

Thus, we can conclusively assign the measured Gaussian line shapes and their strongly temperature-dependent linewidths as intrinsic properties of $\mathrm{Sr}_{3} \mathrm{Ir}_{2} \mathrm{O}_{7}$. Together, these provide direct experimental evidence for a polaronic ground state in $\mathrm{Sr}_{3} \mathrm{Ir}_{2} \mathrm{O}_{7}$, driven by a strong electron-boson coupling. In such a scenario, the quasiparticle residue becomes vanishingly small, and the photoemission measurements are dominated by incoherent excitations at higher binding energies that involve the simultaneous excitation of multiple bosons. Within a simple Franck-Condon broadening (FCB) picture, the measured spectral function is composed of distinct shake-off excitations separated by $\hbar \omega_{0}$, where $\omega_{0}$ is the bosonic mode frequency [Fig. 4(a)]. In the solid state, these individual shakeoff excitations are inherently broadened and so cannot be resolved. However, their intensity distribution, which reflects the overlap of the initial state with different excited final states, follows a Gaussian envelope. This provides a hallmark of a 

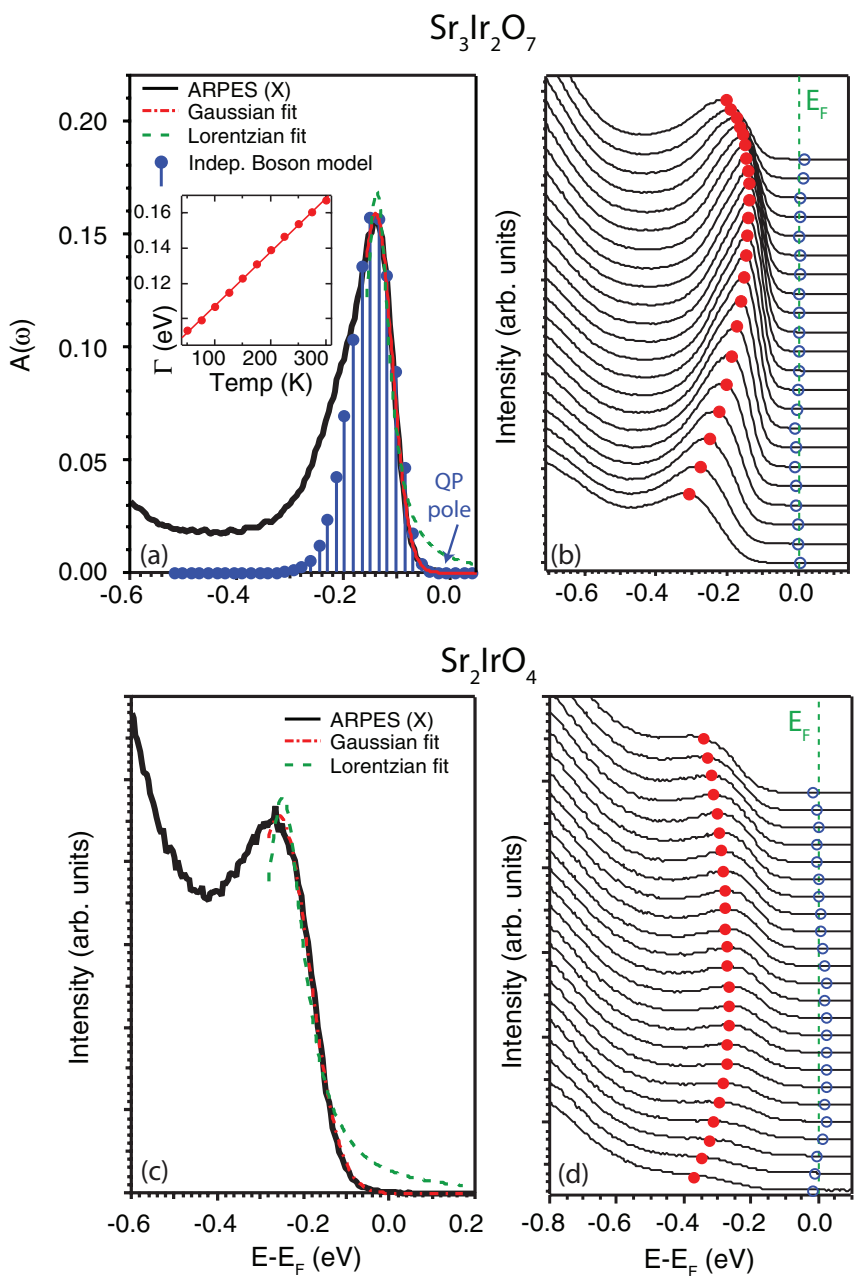

FIG. 4. (Color online) (a) EDC at the $X$ point (black, $h v=$ $40 \mathrm{eV}$ ), with the low-energy side of the peak fit by Gaussian (red dotdashed) and Lorentzian (green dashed) line shapes. An independent boson model calculation $\left[\omega_{0}=0.016 \mathrm{eV}, g=6, T=50 \mathrm{~K}\right.$, with the (zero-temperature) quasiparticle pole $30 \mathrm{meV}$ below the chemical potential] is also shown, in excellent agreement with the measured ARPES data at low binding energies. The inset shows the width of Gaussian fits to the low binding energy half of the peak in the spectral function of equivalent calculations as a function of temperature. (b) EDCs close to the $X$ point. Red closed circles show the peak position of Gaussian fits, $E_{B}$. Blue open circles show $E_{B}+1.76 \Gamma$, where $\Gamma$ is the width of the Gaussian fits. (c),(d) Equivalent measurements (performed at $125 \mathrm{~K}$ to prevent charging) and fits for $\mathrm{Sr}_{2} \mathrm{IrO}_{4}$. In (d), blue open circles are shown at $E_{B}+1.5 \Gamma$.

polaronic system which allows its experimental observation by photoemission, ${ }^{27,36-39}$ without recourse to detailed theoretical treatments or necessitating a microscopic identification of the dominant bosons. With increasing temperature, the linewidth of the Gaussian envelope broadens further, reflecting the presence of thermally excited bosons, ${ }^{40}$ entirely consistent with our measured temperature-dependent linewidths [Figs. 3(b) and 3(c)]. Indeed, these are quantitatively well described by a simple FCB model ${ }^{41}$ From unconstrained fits of the linewidth, $\Gamma(T)$, we find a soft bosonic mode energy of $\omega_{0} \sim 15 \mathrm{meV}$ and a strong coupling constant of $g \sim 5-7$, which would place the system well within the strong coupling regime.
To further validate this picture, we employ these parameters for calculations within the independent boson approximation (IBA).$^{40}$ While this is a simple model that does not attempt to consider the microscopic nature of the bosons responsible, it does yield the generic spectral properties of the polaronic ground state and allows comparison with our experimental data. As shown in Fig. 4(a), the envelope of this model spectral function is in very good agreement with our measured photoemission spectra. ${ }^{42}$ As for the experimental EDCs, the IBA calculations can only be adequately fit by Gaussian line shapes. By explicitly including thermal population effects in our IBA calculations, we extracted the temperature dependence of such Gaussian envelopes, shown inset in Fig. 4(a), which increase almost linearly in width at a rate of $0.3 \mathrm{meV} / \mathrm{K}$. This is in excellent agreement with our direct experimental measurements [Figs. 3(b) and 3(c)], and of the same order (although slightly smaller) than values observed in other polaronic systems. ${ }^{37,41}$

Away from the band top, the linewidth $\Gamma$ increases in direct proportion to the binding energy of the peak in the spectral function $E_{B}$ such that $E_{B}(\mathbf{k}) / \Gamma(\mathbf{k})=1.76 \pm 0.05$ [Fig. 4(b)]. We find similar characteristics of the spectral features in $\mathrm{Sr}_{2} \mathrm{IrO}_{4}$, where we again observe broad Gaussian line shapes [Fig. 4(c)] whose width is proportional to their binding energy [Fig. 4(d), $E_{B}(\mathbf{k}) / \Gamma(\mathbf{k})=1.5 \pm 0.1$ ]. Within the Franck-Condon picture, this implies that the quasiparticle poles have vanishing weight and almost no dispersion, which is consistent with experimental and theoretical findings for other polaronic systems. ${ }^{27,39,43,44}$ We also note that optical conductivity of $\mathrm{Sr}_{2} \mathrm{IrO}_{4}$ was found to show a pronounced dependence on temperature, ${ }^{45}$ very similar to that in the polaronic ground state of the insulating cuprate $\mathrm{La}_{2} \mathrm{CuO}_{4}{ }^{46} \mathrm{Using}$ a simple calculation based on the Franck-Condon scheme proposed here and assuming particle-hole symmetric spectra, our ARPES data reproduce the $\alpha$ peaks measured in optical conductivity ${ }^{1,3}$ of both $\mathrm{Sr}_{3} \mathrm{Ir}_{2} \mathrm{O}_{7}$ and $\mathrm{Sr}_{2} \mathrm{IrO}_{4}$, including their pronounced temperature-dependent broadening. ${ }^{45}$ Moreover, thermal excitation of polaronic carriers was recently proposed as the origin of the Néel transition in $\mathrm{Sr}_{3} \mathrm{Ir}_{2} \mathrm{O}_{7} .{ }^{15}$ These observations all lend further support to our assignment of a polaronic ground state of insulating Ruddlesden-Popper iridates.

Our measured linewidths and binding energies of $\mathrm{Sr}_{2} \mathrm{IrO}_{4}$ are both larger than in the bilayer compound, reflecting a more robustly insulating ground state and stronger electron-boson coupling than in $\mathrm{Sr}_{3} \mathrm{Ir}_{2} \mathrm{O}_{7}$. The microscopic origin of the bosonic mode in either compound, however, remains an open question. While it is tempting to ascribe it to a phonon due to the strong temperature dependence of the measured spectral features, the mode energies obtained from our fits are significantly lower than the dominant phonon branches. ${ }^{47}$ The strong spin-orbit coupling of these compounds is further expected to effectively couple lattice and spin excitations. However, a large magnon gap has previously been reported for $\mathrm{Sr}_{3} \mathrm{Ir}_{2} \mathrm{O}_{7} .{ }^{15}$ If magnetic excitations do play a dominant role, this would imply the presence of a previously unresolved soft magnetic mode in $\mathrm{Sr}_{3} \mathrm{Ir}_{2} \mathrm{O}_{7}$. A complete identification of the relevant degrees of freedom, and their interplay, will therefore require further dedicated theoretical and experimental study. Irrespective of this, however, our observations indicate that the quasiparticle poles are located much closer to the chemical 
potential than would be assumed from conventional interpretations of photoemission spectra within a weakly interacting band picture. Thus, the spectroscopic band gap is much larger than the underlying quasiparticle gap, and magnetic and/or lattice fluctuations play an essential role in stabilizing the insulating nature of layered $5 d$ iridates.

In conclusion, we have characterized the low-energy electronic structure of the bilayer iridate $\mathrm{Sr}_{3} \mathrm{Ir}_{2} \mathrm{O}_{7}$ from temperature-dependent angle-resolved photoemission. At low temperatures, we find a fully gapped insulator, consistent with the nearly exponential temperature dependence of its resistivity, with narrow bandwidths and a small charge gap across the full Brillouin zone. The low-energy spectral features broaden substantially with increasing temperature, a direct experimental signature of strong electron-boson coupling, and exhibit pronounced Gaussian line shapes. We find similar spectral features in the single-layer sister compound $\mathrm{Sr}_{2} \mathrm{IrO}_{4}$, indicative of a Franck-Condon broadening where the simultaneous excitation of multiple bosons dominates the spectral function through incoherent shake-off excitations. Together, these observations constitute the experimental discovery of a polaronic ground state in the unconventional "Mott" state of insulating Ruddlesden-Popper iridates.

This work was supported by the ERC, the UK EPSRC, the AFOSR (FA9550-12-1-0335) and the Grant-in-Aid for Scientific Research (S) (Grant No. 24224010). The research was carried out in part at the Stanford Synchrotron Radiation Lightsource, a Directorate of SLAC National Accelerator Laboratory, and an Office of Science User Facility operated for the US Department of Energy, Office of Science, by Stanford University. *philip.king@st-andrews.ac.uk

${ }^{\dagger}$ felix.baumberger@unige.ch

${ }^{1}$ B. J. Kim, H. Jin, S. J. Moon, J.-Y. Kim, B.-G. Park, C. S. Leem, J. Yu, T. W. Noh, C. Kim, S.-J. Oh, J.-H. Park, V. Durairaj, G. Cao, and E. Rotenberg, Phys. Rev. Lett. 101, 076402 (2008).

${ }^{2}$ B. J. Kim, H. Ohsumi, T. Komesu, S. Sakai, T. Morita, H. Takagi, and T. Arima, Science 323, 1329 (2009).

${ }^{3}$ S. J. Moon, H. Jin, K. W. Kim, W. S. Choi, Y. S. Lee, J. Yu, G. Cao, A. Sumi, H. Funakubo, C. Bernhard, and T. W. Noh, Phys. Rev. Lett. 101, 226402 (2008).

${ }^{4}$ X. Wan, A. M. Turner, A. Vishwanath, and S. Y. Savrasov, Phys. Rev. B 83, 205101 (2011).

${ }^{5}$ A. Shitade, H. Katsura, J. Kuneš, X.-L. Qi, S.-C. Zhang, and N. Nagaosa, Phys. Rev. Lett. 102, 256403 (2009).

${ }^{6}$ D. Pesin and L. Balents, Nat. Phys. 6, 376 (2010).

${ }^{7}$ D. Xiao, W. Zhu, Y. Ran, N. Nagaosa, and S. Okamoto, Nat. Commun. 2, 596 (2011).

${ }^{8}$ B.-J. Yang and Y. B. Kim, Phys. Rev. B 82, 085111 (2010).

${ }^{9}$ Y.-Z. You, I. Kimchi, and A. Vishwanath, Phys. Rev. B 86, 085145 (2012).

${ }^{10}$ F. Wang and T. Senthil, Phys. Rev. Lett. 106, 136402 (2011).

${ }^{11}$ M. K. Crawford, M. A. Subramanian, R. L. Harlow, J. A. FernandezBaca, Z. R. Wang, and D. C. Johnston, Phys. Rev. B 49, 9198 (1994).

${ }^{12}$ G. Cao, Y. Xin, C. S. Alexander, J. E. Crow, P. Schlottmann, M. K. Crawford, R. L. Harlow, and W. Marshall, Phys. Rev. B 66, 214412 (2002).

${ }^{13}$ I. Franke, P. J. Baker, S. J. Blundell, T. Lancaster, W. Hayes, F. L. Pratt, and G. Cao, Phys. Rev. B 83, 094416 (2011).

${ }^{14}$ J. Kim, D. Casa, M. H. Upton, T. Gog, Y.-J. Kim, J. F. Mitchell, M. van Veenendaal, M. Daghofer, J. van den Brink, G. Khaliullin, and B. J. Kim, Phys. Rev. Lett. 108, 177003 (2012).

${ }^{15}$ J. Kim, A. H. Said, D. Casa, M. H. Upton, T. Gog, M. Daghofer, G. Jackeli, J. van den Brink, G. Khaliullin, and B. J. Kim, Phys. Rev. Lett. 109, 157402 (2012).

${ }^{16}$ S. Boseggia, R. Springell, H. C. Walker, A. T. Boothroyd, D. Prabhakaran, D. Wermeille, L. Bouchenoire, S. P. Collins, and D. F. McMorrow, Phys. Rev. B 85, 184432 (2012).

${ }^{17}$ C. Dhital, S. Khadka, Z. Yamani, C. de la Cruz, T. C. Hogan, S. M. Disseler, M. Pokharel, K. C. Lukas, W. Tian, C. P. Opeil, Z. Wang, and S. D. Wilson, Phys. Rev. B 86, 100401(R) (2012).
${ }^{18}$ J. W. Kim, Y. Choi, J. Kim, J. F. Mitchell, G. Jackeli, M. Daghofer, J. van den Brink, G. Khaliullin, and B. J. Kim, Phys. Rev. Lett. 109, 037204 (2012).

${ }^{19}$ S. Fujiyama, K. Ohashi, H. Ohsumi, K. Sugimoto, T. Takayama, T. Komesu, M. Takata, T. Arima, and H. Takagi, Phys. Rev. B 86, 174414 (2012)

${ }^{20}$ F. Ye, S. Chi, B. C. Chakoumakos, J. A. Fernandez-Baca, T. Qi, and G. Cao, Phys. Rev. B 87, 140406(R) (2013).

${ }^{21}$ C. Martins, M. Aichhorn, L. Vaugier, and S. Biermann, Phys. Rev. Lett. 107, 266404 (2011).

${ }^{22}$ X. Liu, V. M. Katukuri, L. Hozoi, W.-G. Yin, M. P. M. Dean, M. H. Upton, J. Kim, D. Casa, A. Said, T. Gog, T. F. Qi, G. Cao, A. M. Tsvelik, J. van den Brink, and J. P. Hill, Phys. Rev. Lett. 109, 157401 (2012).

${ }^{23}$ H. Gretarsson, J. P. Clancy, X. Liu, J. P. Hill, E. Bozin, Y. Singh, S. Manni, P. Gegenwart, J. Kim, A. H. Said, D. Casa, T. Gog, M. H. Upton, H.-S. Kim, J. Yu, V. M. Katukuri, L. Hozoi, J. van den Brink, and Y.-J. Kim, Phys. Rev. Lett. 110, 076402 (2013).

${ }^{24}$ R. Arita, J. Kuneš, A. V. Kozhevnikov, A. G. Eguiluz, and M. Imada, Phys. Rev. Lett. 108, 086403 (2012).

${ }^{25}$ D. Hsieh, F. Mahmood, D. H. Torchinsky, G. Cao, and N. Gedik, Phys. Rev. B 86, 035128 (2012).

${ }^{26}$ J.-M. Carter, V. V. Shankar, M. A. Zeb, and H.-Y. Kee, Phys. Rev. B 85, 115105 (2012).

${ }^{27}$ K. M. Shen, F. Ronning, D. H. Lu, W. S. Lee, N. J. C. Ingle, W. Meevasana, F. Baumberger, A. Damascelli, N. P. Armitage, L. L. Miller, Y. Kohsaka, M. Azuma, M. Takano, H. Takagi, and Z.-X. Shen, Phys. Rev. Lett. 93, 267002 (2004).

${ }^{28}$ D. S. Dessau, T. Saitoh, C.-H. Park, Z.-X. Shen, P. Villella, N. Hamada, Y. Moritomo, and Y. Tokura, Phys. Rev. Lett. 81, 192 (1998).

${ }^{29}$ After completion of this work, we became aware of two related studies on $\mathrm{Sr}_{3} \mathrm{Ir}_{2} \mathrm{O}_{7}$ (Refs. 30 and 31). Our measurements of band dispersions are qualitatively consistent with these reports. However, unlike in Ref. 30, we do not find a large suppression of the spectral weight of near- $E_{F}$ states (although we do observe significant photon-energy dependence of their matrix elements), permitting the detailed line-shape and temperature-dependent study that we perform here. 
${ }^{30}$ B. M. Wojek, M. H. Berntsen, S. Boseggia, A. T. Boothroyd, D. Prabhakaran, D. F. McMorrow, H. M. Ronnow, J. Chang, and O. Tjernberg, J. Phys.: Condens. Matter 24, 415602 (2012).

${ }^{31}$ Q. Wang, Y. Cao, J. A. Waugh, S. R. Park, T. F. Qi, O. B. Korneta, G. Cao, and D. S. Dessau, arXiv:1210.4141.

${ }^{32}$ Indeed, we find very little dispersion $(\lesssim 30 \mathrm{meV})$ of the low-lying excitations at the $X$ point when spanning three full Brillouin zones along the $k_{z}$ direction.

${ }^{33}$ R. Comin, G. Levy, B. Ludbrook, Z.-H. Zhu, C. N. Veenstra, J. A. Rosen, Y. Singh, P. Gegenwart, D. Stricker, J. N. Hancock, D. van der Marel, I. S. Elfimov, A. Damascelli, Phys. Rev. Lett. 109, 266406 (2012).

${ }^{34}$ H. Kuriyama, J. Matsuno, S. Niitaka, M. Uchida, D. Hashizume, A. Nakao, K. Sugimoto, H. Ohsumi, M. Takata, and H. Takagi, Appl. Phys. Lett. 96, 182103 (2010).

${ }^{35}$ F. Baumberger, N. J. C. Ingle, W. Meevasana, K. M. Shen, D. H. Lu, R. S. Perry, A. P. Mackenzie, Z. Hussain, D. J. Singh, and Z.-X. Shen, Phys. Rev. Lett. 96, 246402 (2006).

${ }^{36}$ L. Perfetti, H. Berger, A. Reginelli, L. Degiorgi, H. Höchst, J. Voit, G. Margaritondo, and M. Grioni, Phys. Rev. Lett. 87, 216404 (2001).

${ }^{37}$ K. M. Shen, F. Ronning, W. Meevasana, D. H. Lu, N. J. C. Ingle, F. Baumberger, W. S. Lee, L. L. Miller, Y. Kohsaka, M. Azuma, M. Takano, H. Takagi, and Z.-X. Shen, Phys. Rev. B 75, 075115 (2007).
${ }^{38}$ L. A. Cardenas, Y. Fagot-Revurat, L. Moreau, B. Kierren, and D. Malterre, Phys. Rev. Lett. 103, 046804 (2009).

${ }^{39}$ A. Tamai, A. P. Seitsonen, T. Greber, and J. Osterwalder, Phys. Rev. B 74, 085407 (2006).

${ }^{40}$ G. D. Mahan, Many-Particle Physics, 3rd ed. (Kluwer Academic, Dordrecht, The Netherlands, 2000).

${ }^{41}$ P. H. Citrin, P. Eisenberger, and D. R. Hamann, Phys. Rev. Lett. 33, 965 (1974).

${ }^{42} \mathrm{~A}$ shoulder arising from a second poorly resolved peak in the EDC at higher binding energies is not included in our model, explaining the deviations at higher binding energy.

${ }^{43}$ A. S. Mishchenko and N. Nagaosa, Phys. Rev. Lett. 93, 036402 (2004).

${ }^{44}$ O. Rösch, O. Gunnarsson, X. J. Zhou, T. Yoshida, T. Sasagawa, A. Fujimori, Z. Hussain, Z.-X. Shen, and S. Uchida, Phys. Rev. Lett. 95, 227002 (2005)

${ }^{45}$ S. J. Moon, H. Jin, W. S. Choi, J. S. Lee, S. S. A. Seo, J. Yu, G. Cao, T. W. Noh, and Y. S. Lee, Phys. Rev. B 80, 195110 (2009).

${ }^{46}$ J. P. Falck, A. Levy, M. A. Kastner, and R. J. Birgeneau, Phys. Rev. Lett. 69, 1109 (1992).

${ }^{47}$ M. F. Cetin, P. Lemmens, V. Gnezdilov, D. Wulferding, D. Menzel, T. Takayama, K. Ohashi, and H. Takagi, Phys. Rev. B 85, 195148 (2012). 